\title{
Statistics on ordered partitions of sets
}

\author{
Einar Steingrímsson \\ Department of Computer and Information Sciences \\ University of Strathclyde \\ Glasgow, UK \\ einar@alum.mit.edu
}

Keywords: Ordered set partitions, q-Stirling numbers, permutation statistics.

\section{Prologue}

This paper was first made public on my webpage at Chalmers University of Technology in 2001, after it had been rejected by two journals. It was first posted on the arXiv in 2006 [18, with (trivial) updates in 2007 and 2014.

The reason it is being published now is to put it more formally on the record, as it has been cited in several papers, dealing both directly with some of the conjectures in the paper and in work in other areas. In a series of papers, Ksavrelof and Zeng [12, Ishikawa, Kasraoui and Zeng [9, 8] and Kasraoui and Zeng [10] proved all the conjectures in this paper, the last of these papers giving combinatorial proofs. Also, Remmel and Wilson [15] solved the problem that was the original motivation for the present paper, namely finding a bijective proof of Proposition 2 here, due to Zeng and Zhang 22, thus "explaining" combinatorially this strong connection between ordered set partitions and permutations. Wilson then extended this correspondence to multiset partitions and descent-starred permutations [21].

The statistic ROS defined here also has some bearing on the Delta Conjecture of Haglund, Remmel and Wilson [6], and played a role in Haglund, Rhoades and Shimozono's paper on that conjecture and generalized coinvariant algebras [7].

Apart from this Prologue, the deletion of a now obsolete footnote and the updating of references, the content of the paper is the same as in the original version.

\section{INTRODUCTION}

The Stirling numbers of the second kind, $S(n, k)$, which count the partitions of an $n$-element set into $k$ blocks, have been much studied. Their $q$-analog $S_{q}(n, k)$, the $q$-Stirling numbers of the second kind, can be defined by $S_{q}(n, k)=0$ if $k>n$ or $k<0$ and, for $n \geq k \geq 0$, by the identity

$$
[k] ! \cdot S_{q}(n, k)=\sum_{i}(-1)^{i} \cdot\left[\begin{array}{c}
k \\
i
\end{array}\right] \cdot q^{\left(\begin{array}{c}
i \\
2
\end{array}\right)} \cdot[k-i]^{n},
$$

where

$$
[k]=1+q+\cdots+q^{k-1}, \quad[k] !=[k][k-1] \cdots[1], \quad \text { and } \quad\left[\begin{array}{l}
n \\
k
\end{array}\right]=\frac{[n] !}{[k] ! \cdot[n-k] !} .
$$

Here, as in the remainder of the paper, sums are taken to be over all integers, unless explicitly stated otherwise.

There is an alternative definition of the $q$-Stirling numbers. Those numbers, sometimes denoted $\tilde{S}_{q}(n, k)$, are related to $S_{q}(n, k)$ by $S_{q}(n, k)=q^{\left(\begin{array}{c}k \\ 2\end{array}\right)} \tilde{S}_{q}(n, k)$. In fact, the factor $q^{\left(\begin{array}{c}k \\ 2\end{array}\right)}$ is explicit in some of our statistics, indicating that they essentially correspond to $\tilde{S}_{q}(n, k)$, but as this factor does not appear transparently in some other statistics we take (10) above as our definition.

*Part of this research was carried out while the author was visiting Université Louis-Pasteur in Strasbourg in 1996, supported by the EU Network in Algebraic Combinatorics. 
There are several ways to define $S_{q}(n, k)$ combinatorially, most of them based on statistics on set partitions. Perhaps the simplest of these statistics (in terms of definition) is the one due to Milne [13. It can be defined as follows: Given a partition of the set $\{1,2, \ldots, n\}$ into $k$ blocks, order the blocks in the standard way, that is, by increasing least element. Let $b_{i}$ be the size of the $i$ th block in this ordering. Milne's statistic, which we call MIL, is then defined to be $b_{2}+2 b_{3}+\cdots+$ $(k-1) b_{k}$. As an example, $\operatorname{MIL}(14-238-5-67)=1 \cdot 3+2 \cdot 1+3 \cdot 2=11$.

The following lemma is easily proved from identity (10) or from the definition of MIL given above.

Lemma 1. The numbers $S_{q}(n, k)$ satisfy the recurrence

$$
S_{q}(n, k)=q^{k-1} \cdot S_{q}(n-1, k-1)+[k] \cdot S_{q}(n-1, k) .
$$

Many authors have studied statistics on set partitions with distribution given by the numbers $S_{q}(n, k)$. Apart from the paper by Milne 13, who seems to have pioneered the study of partition statistics whose distribution is given by the $q$ Stirling numbers, we mention [3, 5, 16, 19, 20]. Of these, the paper by Wachs and White [19] is the most comprehensive. Also, there is a substantial literature on various statistics on set partitions restricted to so-called non-crossing partitions. See for example [1, 14, 17, 20].

In the present paper we study statistics on ordered partitions of sets, that is, set partitions where the blocks of a partition are ordered arbitrarily. The original motivation of this study is an identity relating $q$-Stirling numbers of the second kind to $q$-Eulerian numbers (see Proposition 2 below). The Eulerian number $A(n, k)$ counts permutations in the symmetric group $\mathcal{S}_{n}$ with $k$ descents. A descent in a permutation $p=a_{1} a_{2} \cdots a_{n}$ is an $i$ such that $a_{i}>a_{i+1}$. There is a basic identity relating the $S(n, k)$ 's and the $A(n, k)$ 's, namely

$$
k ! \cdot S(n, k)=\sum_{i}\left(\begin{array}{l}
n-i \\
k-i
\end{array}\right) \cdot A(n, i-1),
$$

which is easily proved combinatorially. The $q$-analog of the Eulerian numbers is the MAJ-statistic, defined as the sum of the descents in a permutation. As an example, the permutation $p=5261743$ has descents $1,3,5$ and 6 , and thus MAJ $p=$ $1+3+5+6=15$. We denote by $A_{q}(n, k)$ the distribution of permutations with $k$ descents according to MAJ, that is,

$$
A_{q}(n, k)=\sum_{p \in \mathcal{S}_{n}^{k}} q^{\mathrm{MAJ} p}
$$

where $\mathcal{S}_{n}^{k}$ is the set of permutations in $\mathcal{S}_{n}$ with exactly $k$ descents.

The $q$-analog of identity (3) was derived by Zeng and Zhang [22], using analytic methods, and goes as follows.

Proposition 2 (Zeng and Zhang[22, Proposition 4.6]). For all $n$ and $k$ with $0 \leq$ $k \leq n$ we have

$$
[k] ! \cdot S_{q}(n, k)=\sum_{i} q^{k(k-i)} \cdot\left[\begin{array}{l}
n-i \\
k-i
\end{array}\right] \cdot A_{q}(n, i-1) .
$$

The distribution of the bistatistic (des, MAJ) on the symmetric group $\mathcal{S}_{n}$, that is, the sum of $A_{q}(n, k)$ over all $k$, has been thoroughly studied. This statistic, together with all other bistatistics with the same distribution, is said to be EulerMahonian (the number of descents is an Eulerian statistic and MAJ is a Mahonian statistic). Because of the relation between the $q$-Eulerian numbers and the $q$-Stirling numbers encoded by identity (4), we will refer to statistics on ordered partitions of sets with the distribution $[k] ! \cdot S_{q}(n, k)$ as Euler-Mahonian, and we will even 
call Euler-Mahonian those statistics on (unordered) partitions of sets that have the distribution $S_{q}(n, k)$.

As mentioned above, the original motivation of this paper was the identity (44). We have found several statistics, defined on ordered partitions of sets, whose distribution we conjecture to be that given by either side of (4). Two of these we prove to have this distribution, but in neither case have we been able to find a bijective proof. The obvious combinatorial proof of identity (3) does not generalize to the $q$-analog case of identity (44) in any straightforward way using the known statistics on set partitions. Thus, identity (4) still lacks a combinatorial proof.

Now, given any statistic $S$ with the distribution $S_{q}(n, k)$ on $\mathcal{P}_{n}^{k}$ we could define a composite statistic STAT $=S+T$ on $\mathcal{O P}_{n}^{k}$ by computing $S$ on the standard ordering of the blocks of $\pi \in \mathcal{O} \mathcal{P}_{n}^{k}$ and letting $T$ be any Mahonian permutation statistic (such as INV, the number of inversions, or MAJ) computed on the permutation induced by the ordering of the blocks of $\pi$, since the Mahonian permutation statistics have distribution $[k]$ ! on $\mathcal{S}_{k}$. Thus, we would be computing two separate statistics and effectively ignoring the ordering of the blocks for one of the statistics. However, we know of no such statistic that lends itself to a combinatorial proof of identity (4).

In fact, we are aware of only one statistic defined on set partitions that is independent of the ordering of the blocks, and thus can be combined with any Mahonian permutation statistic on the permutation of the blocks to obtain a statistic with distribution $[k] ! S_{q}(n, k)$ on $\mathcal{O P}_{n}^{k}$. This is the intertwining number of Ehrenborg and Readdy [3, §6], an interesting statistic with properties quite different from those considered here. But, since we don't make any use of this statistic we omit its definition.

Although we have not come up with a bijective proof of (4) we have found a statistic, which we call bmajmiL (see Definition 17), defined on ordered partitions, whose distribution is given by $[k] ! \cdot S_{q}(n, k)$. However, our proof consists of constructing a bijection between ordered partitions of $\{1,2, \cdots, n\}$ with $k$ blocks and the set of permutations with at most $n-k$ descents, marked in certain ways, showing that the statistic in question has the distribution given by the right hand side of (41). We also show that a straightforward generalization of one of the statistics by Wachs and White [19] has distribution $[k] ! \cdot S_{q}(n, k)$.

We have also found several other statistics that we conjecture to have the distribution $[k] ! \cdot S_{q}(n, k)$. All of these conjectures have been verified by computer for $n \leq 11$, so it seems unlikely that they could be wrong.

It is somewhat intriguing that the new statistics introduced are all sums of two statistics, where one is essentially one of two Mahonian permutation statistics, but defined on a certain partial ordering of the blocks of a partition.

It should also be mentioned that the ordered partitions studied here have recently been treated by Krob, Latapy, Novelli, Phan and Schwer [11, under the name of pseudo-permutations. Their results are quite different from ours, but it seems likely that some connections will emerge.

\section{Generalizations of KnOwn statistics}

Let $\mathcal{P}_{n}^{k}$ be the set of (unordered) partitions of the set $\{1,2, \ldots, n\}$ with $k$ blocks and let $\mathcal{O P}_{n}^{k}$ be the set of ordered partitions of the set $\{1,2, \ldots, n\}$ with $k$ blocks. We now introduce several statistics on $\mathcal{O P}_{n}^{k}$ that are generalizations of known statistics on $\mathcal{P}_{n}^{k}$.

So far, most known statistics on $\mathcal{P}_{n}^{k}$ can be defined in terms of inversions between the letters (integers) in a partition $\pi$ and the openers and closers of the blocks of $\pi$. The opener of a block is its least element and the closer is its greatest element. 
For example, the partition $\pi=136-27-4-58$ has openers $1,2,4$ and 5 and closers $6,7,4$ and 8 .

On unordered partitions we always assume, when referring to the ordering of the blocks of a partition, that the blocks are written in increasing order of their respective openers. We call this the standard ordering.

One statistic whose distribution on $\mathcal{P}_{n}^{k}$ is $\tilde{S}_{q}(n, k)$ is the sum $\operatorname{ros} \pi=\sum_{i} \operatorname{ros}_{i} \pi$ where $\operatorname{ros}_{i} \pi$ is the number of openers in $\pi$ that are smaller than $i$ and that belong to blocks to the right of the block containing $i$ (the name ros is of course an abbreviation of "right opener smaller"). For example, the values of $\operatorname{ros}_{i}$ for the partition $\pi=136-27-4-58$ are $0,1,3,0,2,0,0,0$ (written in the order in which the letters appear in $\pi$ ), so $\operatorname{ros} \pi=6$.

We now define ten partition statistics. Four of these were defined by Wachs and White [19, although their treatment was in terms of restricted growth functions, a different way of representing partitions. Another four of our statistics are mirror images of the aforementioned ones that contribute nothing new in the case of unordered partitions. The last two statistics, essentially defined by Foata and Zeilberger 4 for permutations, are in fact each equal to the difference of two of the first eight statistics. However, they are useful to define and they also make clear the similarity between some of our partition statistics and known permutation statistics.

Definition 3. Given a partition $\pi \in \mathcal{O P}_{n}^{k}$, let open $\pi$ and $\operatorname{clos} \pi$ be the set of openers and closers, respectively, of $\pi$. Let $\operatorname{block}(i)$ be the number (counting from the left) of the block containing the letter $i$. We define eight coordinate statistics as follows:

$$
\begin{aligned}
\operatorname{ros}_{i} \pi & =\#\{j \mid i>j, \quad j \in \operatorname{open} \pi, \quad \operatorname{block}(j)>\operatorname{block}(i)\}, \\
\operatorname{rob}_{i} \pi & =\#\{j \mid i<j, \quad j \in \operatorname{open} \pi, \quad \operatorname{block}(j)>\operatorname{block}(i)\}, \\
\operatorname{rcs}_{i} \pi & =\#\{j \mid i>j, \quad j \in \operatorname{clos} \pi, \quad \operatorname{block}(j)>\operatorname{block}(i)\}, \\
\operatorname{rcb}_{i} \pi & =\#\{j \mid i<j, \quad j \in \operatorname{clos} \pi, \quad \operatorname{block}(j)>\operatorname{block}(i)\}, \\
\operatorname{los}_{i} \pi & =\#\{j \mid i>j, \quad j \in \operatorname{open} \pi, \quad \operatorname{block}(j)<\operatorname{block}(i)\}, \\
\operatorname{lob}_{i} \pi & =\#\{j \mid i<j, \quad j \in \operatorname{open} \pi, \quad \operatorname{block}(j)<\operatorname{block}(i)\}, \\
\operatorname{lcs}_{i} \pi & =\#\{j \mid i>j, \quad j \in \operatorname{blos} \pi, \quad \operatorname{block}(j)<\operatorname{block}(i)\}, \\
\operatorname{lcb}_{i} \pi & =\#\{j \mid i<j, \quad j \in \operatorname{clos} \pi, \quad \operatorname{block}(j)<\operatorname{block}(i)\} .
\end{aligned}
$$

Moreover, we let $\mathrm{rsb}_{i}$ be the number of blocks $B$ to the right of the block containing $i$ such that the opener of $B$ is smaller than $i$ and the closer of $B$ is greater than $i$ (rsb is an abbreviation for "right, smaller, bigger"). Also, we define $\mathrm{lsb}_{i}$ in an analogous way, with "right" replaced by "left."

We then set

$$
\operatorname{ros} \pi=\sum_{i} \operatorname{ros}_{i} \pi
$$

and likewise for the remaining nine statistics, i.e. each of rob, rcs, rcb, los, lob, lcs, $\mathrm{lcb}, \mathrm{rsb}, \mathrm{lsb}$ is defined to be the sum over all $i$ of the respective coordinate statistic.

Note that the statistic rsb was first defined, essentially, in [4] (see also [2]) as a "partial statistic" in the definition of the permutation statistic MAK. We will treat a partition statistic analogous to MAK later in this paper.

As an example of the coordinate statistics just defined we give the values of three of them on the ordered partition $\pi=47-3-159-68-2$ :

$\begin{array}{lllllllllllll}\pi= & 4 & 7 & - & 3 & - & 1 & 5 & 9 & - & 6 & 8 & - \\ \operatorname{ros}_{i}: & 3 & 4 & 2 & & 0 & 1 & 2 & & 1 & 1 & 0 \\ \operatorname{lcb}_{i}: & 0 & 0 & 1 & & 2 & 1 & 0 & & 2 & 1 & \\ \operatorname{rsb}_{i}: & 1 & 2 & 1 & 0 & 0 & 0 & & 0 & 0 & 0\end{array}$


On unordered partitions, the statistic MIL mentioned above is in fact equal to los (left opener smaller), since the opener of every block to the left of the block containing a given letter is smaller than that letter. Thus, los has the distribution $S_{q}(n, k)$ on $\mathcal{P}_{n}^{k}$. On the other hand, lob (left opener bigger) is clearly identically zero on $\mathcal{P}_{n}^{k}$.

On ordered partitions the situation is much simpler, since "left" and "right" have equal status, that is, reversing the order of the blocks in an ordered partition turns a left opener into a right opener and likewise for closers. Thus every right statistic is equidistributed with its corresponding left statistic.

Moreover, given a partition $\pi$, let $\pi^{c}$ be the partition obtained by complementing each of the letters in $\pi$, that is, by replacing the letter $i$ by $n+1-i$. It is then easily checked that $\operatorname{rcb} \pi^{c}=\operatorname{ros} \pi$ and that $\operatorname{rcs} \pi^{c}=\operatorname{rob} \pi$. Thus the eight statistics obtained by independently varying left/right, opener/closer and smaller/bigger fall into only two categories when it comes to their distribution on ordered partitions. One of these categories consists of ros, rcb, los and lcb, and the other contains rob, rcs, lcs and lob. Since $\operatorname{rcb}_{i} \geq \operatorname{rob}_{i}$ for all $i$ and any partition $\pi$, it is clear that the statistics in the latter category are "smaller" than those in the first one. Indeed, the "small" statistics do not have a distribution related in any obvious way to the $S_{q}(n, k)$, except that $\mathrm{rob}_{i}$ equals $\mathrm{rcb}_{i}-\mathrm{rsb}_{i}$, which is easily proved as are the analogous identities for rcs, lcs and lob. However, the "small" statistics play a role in some of our new statistics, which consist of combinations of these with yet other statistics.

It should perhaps be mentioned that there is a way to redefine ros in order to "split" it into a partition statistic and a Mahonian statistic on the permutation of the blocks. Namely, $\operatorname{ros}_{i}$, when restricted to the openers of $\pi$, records just the inversions among the openers, and thus the inversion statistic on the permutation of the blocks of $\pi$ (as compared to the standard ordering). However, as mentioned before, we have not been able to exploit this to find a combinatorial proof of Proposition 2 .

The statistic ros has distribution $\tilde{S}_{q}(n, k)$ on $\mathcal{P}_{n}^{k}$ and on $\mathcal{O P}_{n}^{k}$ its distribution is $[k] ! \tilde{S}_{q}(n, k)$. In order to get the distribution $[k] ! S_{q}(n, k)$ we therefore add $\left(\begin{array}{l}k \\ 2\end{array}\right)$, where $k$ is the number of blocks in $\pi$. We call this new statistic ROS, so ROS $\pi=$ $\operatorname{ros} \pi+\left(\begin{array}{l}k \\ 2\end{array}\right)$.

Theorem 4. The statistic ROS is Euler-Mahonian on ordered partitions, that is,

$$
\sum_{\pi \in \mathcal{O} \mathcal{P}_{n}^{k}} q^{\mathrm{ROS} \pi}=[k] ! S_{q}(n, k)
$$

Proof. The proof is by induction on $n$, with $k$ fixed. Suppose first that $\pi$ is a partition in $\mathcal{O P}_{n}^{k}$, such that $n$ is a singleton block. Then $\pi$ can be obtained from a unique partition $\pi^{\prime}$ in $\mathcal{O} \mathcal{P}_{n-1}^{k-1}$, by inserting $n$ as a singleton block in one of the slots between blocks in $\pi^{\prime}$, or before, or after, all the blocks in $\pi$. If $\pi$ is obtained by inserting $n$ right after block $i$ in $\pi^{\prime}$, then there will be $(k-1-i)$ blocks following $n$ so this increases ROS by $k-1-i$. Moreover, since we now have $k$ blocks instead of $k-1$, ROS is additionally increased by $\left(\begin{array}{c}k \\ 2\end{array}\right)-\left(\begin{array}{c}k-1 \\ 2\end{array}\right)=k-1$. Therefore the total increase in $\operatorname{ROS}$ is $(k-1-i)+(k-1)$.

Thus, if $S$ is the set of partitions obtained from the partition $\pi^{\prime}$ by inserting $n$ in the $k$ slots between blocks in $\pi^{\prime}$ or before or after all of the blocks, then we have

$$
\sum_{\pi \in S} q^{\mathrm{ROS} \pi}=q^{\mathrm{ROS} \pi^{\prime}} q^{k-1}\left(1+q+\cdots+q^{k-1}\right)=q^{\mathrm{ROS} \pi^{\prime}} q^{k-1}[k] .
$$

On the other hand, if $n$ is not a singleton block in $\pi$, then $\pi$ can be obtained from a unique partition $\pi^{\prime}$ in $\mathcal{O P} \mathcal{P}_{n-1}^{k}$, by inserting $n$ in one of the blocks in $\pi^{\prime}$. If $n$ is thus inserted into the $i$-th block we increase ROS by $(k-i)$, so the corresponding 
sum, with $T$ defined analogously to $S$ above, is

$$
\sum_{\pi \in T} q^{\mathrm{ROS} \pi}=q^{\mathrm{ROS} \pi^{\prime}}\left(1+q+\cdots+q^{k-1}\right)=q^{\mathrm{ROS} \pi^{\prime}}[k] .
$$

Thus we have, assuming the statement true for $n-1$, that

$$
\begin{aligned}
\sum_{\pi \in \mathcal{O} \mathcal{P}_{n}^{k}} q^{\mathrm{ROS} \pi} & =q^{k-1}[k][k-1] ! S_{q}(n-1, k-1)+[k][k] ! S_{q}(n-1, k) \\
& =[k] !\left(q^{k-1} S_{q}(n-1, k-1)+[k] S_{q}(n-1, k)\right),
\end{aligned}
$$

which, by Lemma 1 equals $[k] ! S_{q}(n, k)$ as desired. The case $n=1$ is trivial (and the case $n=0$ a matter of definition).

Corollary 5. The following statistics are Euler-Mahonian on $\mathcal{O P}_{n}^{k}$ :

$$
\begin{aligned}
\mathrm{RCB} & =\operatorname{rcb}+\left(\begin{array}{l}
k \\
2
\end{array}\right), \\
\mathrm{LOS} & =\operatorname{los}+\left(\begin{array}{l}
k \\
2
\end{array}\right), \\
\mathrm{LCB} & =\operatorname{lcb}+\left(\begin{array}{l}
k \\
2
\end{array}\right) .
\end{aligned}
$$

\section{A NEW EULER-MAHONIAN STATISTIC ON ORDERED PARTITIONS}

We now define a new statistic that has the distribution $[k] ! S_{q}(n, k)$ on $\mathcal{O P}_{n}^{k}$. This statistic, and each of the new statistics we introduce in the next section, is a combination of two statistics $S$ and $T$, where $S$ is a partition statistic that is inspired by a permutation statistic. What is surprising is that the $T$-statistics are permutation statistics computed on the partial ordering of the blocks of a partition defined by setting $B_{i}<B_{j}$ if each letter of $B_{i}$ is smaller than every letter of $B_{j}$. We consider two such permutation statistics on the blocks, corresponding to INV and MAJ, respectively.

Definition 6. Let $\pi$ be an ordered partition in $\mathcal{O} \mathcal{P}_{n}^{k}$ with blocks $B_{1}, B_{2}, \ldots, B_{k}$ and let the partial ordering of the blocks be as defined in the preceding paragraph. We say that $i$ is a block descent in $\pi$ if $B_{i}>B_{i+1}$.

The block major index of $\pi$, denoted bMAJ $\pi$, is the sum of the block descents in $\pi$. A block inversion in $\pi$ is a pair $(i, j)$ such that $i<j$ and $B_{i}>B_{j}$.

The block inversion number of $\pi$ denoted bINV $\pi$, is the number of block inversions in $\pi$.

Moreover, we set cbMAJ $=\left(\begin{array}{l}k \\ 2\end{array}\right)-$ bMAJ and cbINV $=\left(\begin{array}{l}k \\ 2\end{array}\right)-$ bINV.

Note that cbmaJ is the sum of the elements in the complement of the set of block descents in $\pi$, whence the prefixed "c," and likewise for cbinv.

For example, if $\pi=41-96-5-87-32$ (where we write the elements of each block decreasingly to emphasize the block descents) then bINV $\pi=0+2+1+1+0=4$ and bMAJ $\pi=2+4=6$ since the first block is not larger than any other block, the second block is larger than the third and the fifth blocks, and the third and the fourth blocks are both larger than the fifth block.

The new statistic we now introduce is the sum of the statistic bMAJ and the statistic MIL computed on ordered partitions in the same way as for unordered partitions. (Thus, on ordered partitions, MIL is not equal to los as it is on $\mathcal{P}_{n}^{k}$.) 
Definition 7. Let $\pi$ be an ordered partition with blocks $B_{1}, B_{2}, \ldots, B_{k}$ and let $b_{i}$ be the size of block $i$. Then

$$
\operatorname{bmajMIL~} \pi=\operatorname{bMAJ} \pi+\sum_{i}(i-1) b_{i} .
$$

For example, bmajmiL $(41-96-5-87-32)=(2+4)+(1 \cdot 2+2 \cdot 1+3 \cdot 2+4 \cdot 2)=$ $6+18=24$.

Note that MIL is closely related to the MAJ-statistic on permutations. Namely, MAJ of a permutation $p$ can be computed by assigning to each letter in $p$ the number of descents to its right and then summing these numbers. This gives the same result as writing $p$ backwards, cutting it at each non-descent and then computing MIL on the resulting partition. As an example,

$$
\begin{aligned}
\operatorname{MAJ}(28613745) & =3+3+2+1+1+1+0+0 \\
\operatorname{MIL}(54-731-6-82) & =0+0+1+1+1+2+3+3 .
\end{aligned}
$$

In order to prove that bmajmiL has the distribution $[k] ! S_{q}(n, k)$ we need to rewrite identity (2). Recall that $A_{q}(n, k)$ is the polynomial in $q$ whose $i$-th coefficient is the number of permutations in $\mathcal{S}_{n}$ with $k$ descents and MAJ equal to $i$.

Lemma 8. $\quad A_{q}(n, i-1)=q^{n i-\left(\begin{array}{c}n+1 \\ 2\end{array}\right)} \cdot A_{q}(n, n-i)$.

Proof. If we reverse a permutation in $\mathcal{S}_{n}$ with $i-1$ descents, the resulting permutation will have exactly $n-i$ descents. Moreover, it is easy to see that the change in MAJ when reversing a permutation depends only on $n$ and $i$. It is also easy to see that the polynomials $A_{q}(n, k)$ are symmetric (reverse a permutation and complement each letter, i.e., replace $k$ by $n+1-k$ ). Thus it suffices to compute the minimum of MAJ on the set of permutations with $i-1$ descents and on the set of those with $n-i$ descents, respectively, in order to determine the shift in the exponents of $q$ when going from $A_{q}(n, i-1)$ to $A_{q}(n, n-i)$. The difference between these two minima is of course

$$
\sum_{k=1}^{i-1} i-\sum_{k=1}^{n-i} i=\left(\begin{array}{l}
i \\
2
\end{array}\right)-\left(\begin{array}{c}
n-i+1 \\
2
\end{array}\right)=(i \cdot(i-1)-(n-i+1)(n-i)) / 2,
$$

which is easily seen to equal $n i-\left(\begin{array}{c}n+1 \\ 2\end{array}\right)$.

Using Lemma 8 we can now rewrite Proposition 2 in the following form.

\section{Proposition 9 (Equivalent to Proposition 2).}

$$
[k] ! \cdot S_{q}(n, k)=\sum_{i} q^{k(k-i)+n i-\left(\begin{array}{c}
n+1 \\
2
\end{array}\right)} \cdot\left[\begin{array}{l}
n-i \\
k-i
\end{array}\right] \cdot A_{q}(n, n-i) .
$$

It is well-known that the $q$-binomial coefficient $\left[\begin{array}{l}n \\ k\end{array}\right]$ records the inversion statistic on binary words of length $n$ with exactly $k 1$ 's. As an example, $\left[\begin{array}{l}3 \\ 2\end{array}\right]=1+q+q^{2}$, corresponding to the words $011,101,110$, whose number of inversions is zero, one and two, respectively. We will be concerned with inversions in binary words associated to subsets of the set of descents of certain permutations.

The descent blocks of a permutation $p$ are the maximal contiguous decreasing subsequences of $p$. For example, the permutation 521364 has descent blocks $521-$ $3-64$.

Let $p$ be a permutation in $\mathcal{S}_{n}$ with descent blocks $B_{1}, B_{2}, \ldots, B_{i}$. Then $p$ has $n-i$ descents. Pick $k-i$ of these descents and mark them with 1 's and mark the descents not picked with 0 's. Let $w$ be the binary word obtained by reading the 0's and 1's from left to right. We translate the permutation $p$, together with the set of 
chosen descents (coded by $w$ ) into an ordered partition $\pi$ with $k$ blocks by cutting $p$ into its descent blocks and then further cutting the descent blocks at the chosen descents.

As an example, let $p=51-742-6-83$, where we use the dashes to indicate the partition of $p$ into descent blocks. If we pick the descents indicated by vertical bars in $5|1-74| 2-6-83$ then $w=1010$ and the ordered partition obtained from $(p, w)$ is $\pi=5-1-74-2-6-83$. Now MAJ $p=1+3+4+7=15$, INV $w=3$ and bmajmiL $(5-1-74-2-6-83)=(1+4+3+4+10)+(1+3)=26$. Thus, bmajmiL $\pi=$ MAJ $p+\operatorname{INV} w+8$. As it happens, the number 8 here equals $k(k-i)+n i-\left(\begin{array}{c}n+1 \\ 2\end{array}\right)$ (cf. Proposition 9), since we have $n=8, k=6$ (the number of blocks in $\pi$ ) and $i=4$ (the number of descent blocks in $p$ ).

We will now prove that this holds in general, thus showing that the distribution of bmajмiL on $\mathcal{O P}_{n}^{k}$ is given by the right hand side in Proposition 9 .

Theorem 10. The distribution of bmajmiL on $\mathcal{O P}_{n}^{k}$ is

$$
\sum_{i} q^{k(k-i)+n i-\left(\begin{array}{c}
n+1 \\
2
\end{array}\right)} \cdot\left[\begin{array}{l}
n-i \\
k-i
\end{array}\right] \cdot A_{q}(n, n-i) .
$$

Thus, bmajmiL is Euler-Mahonian on ordered partitions.

Proof. The proof is along the lines in the example preceding the theorem. That is, to each pair $(p, w)$, where $p$ is a permutation in $\mathcal{S}_{n}$ with $n-i$ descents and $w$ is a binary word of length $n-i$ with $k-i 1$ 's, we associate a partition $\pi$ by cutting $p$ at each non-descent and at those descents in $p$ that correspond to 1's in $w$. This is clearly a bijective correspondence since each ordered partition in $\mathcal{O P}_{n}^{k}$ gives rise to a unique permutation in $\mathcal{S}_{n}$ with $n-i$ descents, exactly $k-i$ of which are marked (by virtue of occurring between adjacent blocks of $\pi$ ).

We show that the difference bmajMIL $\pi-(\operatorname{INV} w+$ MAJ $p)$ equals the exponent $k(k-i)+n i-\left(\begin{array}{c}n+1 \\ 2\end{array}\right)$ in the sum, which establishes the theorem.

We fix $n$ and $i$, and proceed by induction on $k$. The base case is $k=i$, since $k \geq i$. The induction step consists of two parts, the first of which is a lemma that shows we may restrict our attention to words $w$ whose last letter is a 0 .

(i) If $k=i$, referring to the example preceding the theorem, we have a permutation with no marked descents, so $w$ is the all zero word, whose INV is 0 . Thus we need to show that given a permutation $p$ with $k$ descent blocks, its MAJ differs from bmajMIL of the corresponding ordered partition $\pi$ by the exponent $k(k-i)+n i-\left(\begin{array}{c}n+1 \\ 2\end{array}\right)=0+n(k-1)-\left(\begin{array}{c}n \\ 2\end{array}\right)=n k-\left(\begin{array}{c}n+1 \\ 2\end{array}\right)$.

Let $b_{1}, b_{2}, \cdots, b_{k}$ be the sizes of the descent blocks in $p$. Then

$$
\begin{aligned}
\operatorname{MAJ} p & =\left(\begin{array}{c}
n+1 \\
2
\end{array}\right)-b_{1}-\left(b_{1}+b_{2}\right)-\cdots-\left(b_{1}+b_{2}+\cdots+b_{k}\right) \\
& =\left(\begin{array}{c}
n+1 \\
2
\end{array}\right)-\sum_{i=1}^{k}(k+1-i) b_{i}
\end{aligned}
$$

and, since bMAJ $\pi=0$, we have

$$
\text { bmajмIL } \pi=b_{2}+2 b_{3}+\cdots+(k-1) b_{k}=\sum_{i=1}^{k}(i-1) b_{i} .
$$

Thus

$$
\operatorname{bmajMIL~} \pi-\operatorname{MAJ} p=k \sum b_{i}-\left(\begin{array}{c}
n+1 \\
2
\end{array}\right)=k n-\left(\begin{array}{c}
n+1 \\
2
\end{array}\right),
$$

as desired. 
(ii) We now show that given a permutation $p$ and a binary word $w$ indicating which of the descents in $p$ have been chosen, if we transpose a 0 in $w$ with an adjacent 1 to its right then the change in bmajmIL of the corresponding partition is 1 , which equals the change in INV $w$. (The transposition in $w$ does not, of course, affect MAJ $p$.)

We first consider the case where the 0 and the 1 belong to the same descent block. Then bmajmiL of the corresponding partition also increases by 1 because one letter gets moved from a block to the next one, whereas the number and position of block-descents is unchanged.

If the 0 and 1 belong to different blocks then two letters get moved up one block, but the block descent associated to the 1 is reduced by one. Thus the total increase in bmajmiL is 1 .

(iii) Now we observe what happens when we increase $k$ by 1 . This corresponds to changing one of the 0 's in $w$ to a 1 . By repeated use of (ii) we may assume that the last letter in $w$ is a 0 and that we are changing that 0 to a 1.

The exponent $k(k-i)+n i-\left(\begin{array}{c}n+1 \\ 2\end{array}\right)$ now increases by $2 k+1-i$, but INV decreases by $k-i$, since one inversion is lost for each of the 1 's we had. Thus the total increase on the permutation side is $k+1$.

On the partition side, suppose that the last $j$ blocks in $\pi$ are singletons (and not the block preceding them). Then the 0 that is being changed to a 1 lies between the last two letters in block $k-j$ so the block number of the last letter in block $k-j$ is increased by 1 and the same is true of each of the $j$ letters in the trailing singleton blocks. This contributes an increase of $j+1$ to bmajmiL. Moreover, we introduce a new block descent in position $k-j$. Thus the total increase in bmajmiL is also $k+1$.

\section{Other (CONJectured) Euler-Mahonian statistics}

We now introduce several new statistics on ordered partitions. All of these, like the statistic bmajMIL, are sums of one statistic defined on the elements of the ordered partition and one statistic defined on the partial ordering of the blocks described above.

All the conjectures in this section have been verified by computer for all $n \leq 11$. This is strong evidence, because what could "go wrong" we expect to do so for much smaller $n$.

We start with a preliminary definition of a partition statistic that we call MAK. Namely, we let $\operatorname{clos}_{i}$ be the closer of the $i$-th block in $\pi$ and set

$$
\operatorname{MAK} \pi=\sum_{i}\left(n-\operatorname{clos}_{i}\right)+\operatorname{rsb} \pi .
$$

This statistic is essentially the same as the Mahonian permutation statistic bearing the same name, that was defined by Foata and Zeilberger [4. Namely, let ncl $\pi$ be the sum of the non-closers in $\pi$ and observe that $\sum_{i} \operatorname{clos}_{i}=\left(\begin{array}{c}n+1 \\ 2\end{array}\right)-\operatorname{ncl} \pi$. Then MAK $\pi$ can be rewritten in the following way, assuming $\pi$ has $k$ blocks:

$$
\begin{aligned}
\sum_{i}\left(n-\operatorname{clos}_{i}\right)+\operatorname{rsb} \pi & =k n-\sum_{i} \operatorname{clos}_{i}+\operatorname{rsb} \pi \\
& =k n-\left(\begin{array}{c}
n+1 \\
2
\end{array}\right)+\operatorname{ncl} \pi+\operatorname{rsb} \pi .
\end{aligned}
$$

Now, for $n$ and $k$ fixed, the terms $k n-\left(\begin{array}{c}n+1 \\ 2\end{array}\right)$ only amount to a constant shift of the statistic. What remains is $\operatorname{ncl} \pi+\operatorname{rsb} \pi$ which is equal to MAK $p$, where $p$ is the permutation obtained from $\pi$ by writing the elements of the first block of $\pi$ in decreasing order, then those of the second block in decreasing order and so 
on, assuming that $\pi$ was written in standard form. As an example, the partition $421-63-85-7$ yields the permutation 42163857 . Thus, MAK is, conjecturally, an Euler-Mahonian statistic both on partitions and permutations.

There is another way to rewrite MAK that is also interesting. Namely, let $\mathrm{scl}_{i}$ be the number of closers in $\pi$ that are smaller than the $i$-th letter in $\pi$. Then $\sum_{i}\left(n-\operatorname{clos}_{i}\right)=\sum_{i} \operatorname{scl}_{i}$. Moreover, since no letter is larger than the closer of its own block, we have that $\operatorname{scl}_{i}=\operatorname{lcs}_{i}+\mathrm{rcs}_{i}$. Thus, as we also have rsb $=$ ros $-\mathrm{rcs}$, this entails that

$$
\begin{aligned}
\text { MAK } & =\sum_{i}\left(n-\operatorname{clos}_{i}\right)+\mathrm{rsb}=\sum_{i} \operatorname{scl}_{i}+\mathrm{rsb} \\
& =\mathrm{lcs}+\mathrm{rcs}+\mathrm{rsb}=\mathrm{lcs}+\mathrm{rcs}+(\mathrm{ros}-\mathrm{rcs})=\mathrm{lcs}+\mathrm{ros} .
\end{aligned}
$$

On ordered partitions the statistic $\mathrm{MAK}^{\prime}=\mathrm{lob}+\mathrm{rcb}$ is clearly equidistributed with MAK, which can be seen by complementing the letters of a partition. Moreover, by the same reasoning, the statistics $n(k-1)-(\mathrm{lcb}+\mathrm{rob})$ and $n(k-1)-(\operatorname{los}+\mathrm{rcs})$ are equidistributed with each other.

For clarity we now write up the definitions of these four statistics.

\section{Definition 11.}

$$
\begin{aligned}
\text { MAK } & =\text { lcs }+ \text { ros }, \\
\text { MAK }^{\prime} & =\mathrm{lob}+\mathrm{rcb}, \\
\ell \mathrm{MAK}^{\prime} & =n(k-1)-(\mathrm{los}+\mathrm{rcs}) \\
\ell \mathrm{MAK}^{\prime} & =n(k-1)-(\mathrm{lcb}+\mathrm{rob}) .
\end{aligned}
$$

We conjecture that each of the above statistics can be paired with either bINV or bMAJ to yield an Euler-Mahonian statistic on ordered partitions.

Conjecture 12. Each of the following eight statistics is Euler-Mahonian on $\mathcal{O P}_{n}^{k}$.

$$
\begin{array}{llll}
\text { MAK + bMAJ, } & \text { MAK' }^{\prime} \text { bMAJ }, & \ell \text { MAK + bMAJ, } & \ell \mathrm{MAK}^{\prime}+\text { bMAJ }, \\
\text { MAK + bINV, } & \text { MAK }+ \text { bINV, } & \ell \text { MAK + bINV, } & \ell \text { MAK }^{\prime}+\text { bINV } .
\end{array}
$$

Since both bINV and bMAJ vanish on $\mathcal{P}_{n}^{k}$ the above eight statistics reduce to the statistics in Definition 11 when restricted to $\mathcal{P}_{n}^{k}$. It was pointed out by an anonymous referee that they are all Euler-Mahonian on $\mathcal{P}_{n}^{k}$, that MAK ${ }^{\prime}$ and $\ell$ MAK are both equal (as functions) to RCB (see Theorem 4), and that MAK and $\ell \mathrm{MAK}^{\prime}$ are equal, and can be shown to be Euler-Mahonian using a bijection in Section 3 of $[20]$.

The statistics in Definition 11 were also shown to be Euler-Mahonian by Gérald Ksavrelof and Jiang Zeng [12]. In fact, they proved more. Namely, given any partition $\pi=B_{1}-B_{2}-\cdots-B_{k}$ of $[n]$ into $k$ blocks and $d$ with $0 \leq d \leq k$, define

$$
\operatorname{MAK}_{d} \pi=\operatorname{MAK} \pi+k-d-\#\left\{a \in B_{j} \mid j>d, a>\operatorname{clos}_{d}\right\} .
$$

Then they prove that $\mathrm{MAK}_{d}$ is Euler-Mahonian on $P_{n}^{k}$. Note that $\mathrm{MAK}_{k}=\mathrm{MAK}$

The last new statistic we introduce is not based on any Mahonian permutation statistic, but rather on the coordinate statistic lsb introduced in Definition 3. We could of course make an analogous definition for rsb, which would require a "right" analog of cbMAJ and cbinv.

Conjecture 13. The statistics

$$
\begin{aligned}
\text { cmajLSB } & =\operatorname{lsb}+\operatorname{cbmAJ}+\left(\begin{array}{l}
k \\
2
\end{array}\right), \\
\text { cinvLSB } & =\operatorname{lsb}+\operatorname{cbINV}+\left(\begin{array}{l}
k \\
2
\end{array}\right)
\end{aligned}
$$


are Euler-Mahonian on $\mathcal{O} \mathcal{P}_{n}^{k}$.

Note that when we restrict to $\mathcal{P}_{n}^{k}$, both cmajLSB and cinvLSB reduce to lsb $+\left(\begin{array}{l}k \\ 2\end{array}\right)$. On $\mathcal{P}_{n}^{k}$ the statistic lsb is equal to lcb, because each block to the left of the block containing a given letter $i$ has an opener that is smaller than $i$. Thus, by Corollary 5 , lsb $+\left(\begin{array}{c}k \\ 2\end{array}\right)$ is Euler-Mahonian on $\mathcal{P}_{n}^{k}$.

Finally we point out an equivalent way of formulating the conjecture that MAK + bMAJ and MAK + bINV are Euler-Mahonian. This formulation gives a simpler form for the sum in Proposition 9 because the shift in MAK mentioned above can be factored out from that sum in a way that allows us to eliminate the power of $q$ involved. We use the following lemma, where $\left[\begin{array}{l}a \\ b\end{array}\right]_{q^{-1}}$ is the polynomial in $q^{-1}$ obtained by replacing $q$ with $q^{-1}$ in $\left[\begin{array}{l}a \\ b\end{array}\right]$ :

\section{Lemma 14}

$$
\left[\begin{array}{l}
n-i \\
k-i
\end{array}\right]_{q^{-1}}=q^{(k-n)(k-i)}\left[\begin{array}{l}
n-i \\
k-i
\end{array}\right] .
$$

Proof. It is well known that $\left[\begin{array}{l}a \\ b\end{array}\right]$ is symmetric as a polynomial in $q$. Therefore, since the effect of replacing $q$ by $q^{-1}$ in the polynomial $\left[\begin{array}{l}a \\ b\end{array}\right]$ is to reverse the coefficients and shift the exponents of $q$ in $\left[\begin{array}{l}a \\ b\end{array}\right]$, to prove the lemma we only need to compute the degree of $\left[\begin{array}{c}n-i \\ k-i\end{array}\right]$ as a polynomial in $q$. This degree is $(n-k)(k-i)$.

Let now $M_{q}(n, k)$ be the distribution of ncl $+\mathrm{rsb}+$ bmaJ on $\mathcal{O P}{ }_{n}^{k}$. Then, by identity (5), $M_{q}(n, k)$ equals the distribution on $\mathcal{O P}_{n}^{k}$ of MAK + bMAJ, divided by

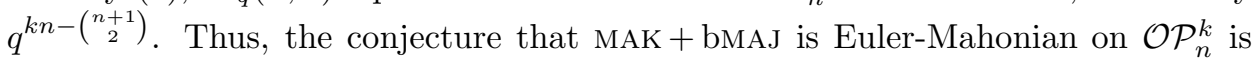
equivalent to

$$
M_{q}(n, k)=q^{\left(\begin{array}{c}
n+1 \\
2
\end{array}\right)-k n} \sum_{i} q^{k(k-i)+n i-\left(\begin{array}{c}
n+1 \\
2
\end{array}\right)} \cdot\left[\begin{array}{l}
n-i \\
k-i
\end{array}\right] \cdot A_{q}(n, n-i) .
$$

Rewriting the power of $q$ in the right hand side and then applying Lemma 14 we obtain the following, which is equivalent to the first part of Conjecture 12.

\section{Conjecture 15.}

$$
M_{q}(n, k)=\sum_{i}\left[\begin{array}{l}
n-i \\
k-i
\end{array}\right]_{q^{-1}} \cdot A_{q}(n, n-i) .
$$

Observe that we could also take $M_{q}(n, k)$ to be the distribution of ncl + rsb + binv. What is perhaps most interesting about Conjecture 15] is the fact that $A_{q}(n, n-i)$ is also the distribution of the permutation statistic MAK on the set of permutations in $\mathcal{S}_{n}$ with exactly $n-i$ descents. (In other words, the bistatistics (des, MAK) and (des, MAJ) have the same distribution on $\mathcal{S}_{n}$.) One might thus hope to find a simple proof of Conjecture 15, since we are computing essentially the same statistic on both sides, but we have been unable to find such a proof.

\section{ACKNOWLEDGMENTS}

My thanks to Jiang Zeng for fruitful discussions in the initial stage of the research presented here, and to the anonymous referee mentioned after Conjecture 12

\section{REFERENCES}

[1] M. Bóna and R. Simion. A self-dual poset on objects counted by the Catalan numbers and a type-B analogue. Discrete Math., 220(1-3):35-49, 2000.

[2] R. J. Clarke, E. Steingrímsson, and J. Zeng. New Euler-Mahonian statistics on permutations and words. Adv. in Appl. Math., 18(3):237-270, 1997. 
[3] R. Ehrenborg and M. Readdy. Juggling and applications to $q$-analogues. In Proceedings of the 6th Conference on Formal Power Series and Algebraic Combinatorics (New Brunswick, NJ, 1994), volume 157, pages 107-125, 1996.

[4] D. Foata and D. Zeilberger. Denert's permutation statistic is indeed Euler-Mahonian. Stud. Appl. Math., 83(1):31-59, 1990.

[5] A. M. Garsia and J. B. Remmel. Q-counting rook configurations and a formula of Frobenius. J. Combin. Theory Ser. A, 41(2):246-275, 1986.

[6] J. Haglund, J. B. Remmel, and A. T. Wilson. The delta conjecture. Trans. Amer. Math. Soc., 370(6):4029-4057, 2018.

[7] J. Haglund, B. Rhoades, and M. Shimozono. Ordered set partitions, generalized coinvariant algebras, and the delta conjecture. Adv. Math., 329:851-915, 2018.

[8] M. Ishikawa, A. Kasraoui, and J. Zeng. Euler-Mahonian statistics on ordered partitions and Steingrímsson's conjecture - a survey. In Combinatorial representation theory and related topics, RIMS Kôkyûroku Bessatsu, B8, pages 99-113. Res. Inst. Math. Sci. (RIMS), Kyoto, 2008.

[9] M. Ishikawa, A. Kasraoui, and J. Zeng. Euler-Mahonian statistics on ordered set partitions. SIAM J. Discrete Math., 22(3):1105-1137, 2008.

[10] A. Kasraoui and J. Zeng. Euler-Mahonian statistics on ordered set partitions. II. J. Combin. Theory Ser. A, 116(3):539-563, 2009.

[11] D. Krob, M. Latapy, J.-C. Novelli, H. D. Phan, and S. Schwer. Pseudo-permutations I: First combinatorial and lattice properties, 2000.

[12] G. Ksavrelof and J. Zeng. Nouvelles statistiques de partitions pour les $q$-nombres de Stirling de seconde espèce. Discrete Math., 256(3):743-758, 2002. LaCIM 2000 Conference on Combinatorics, Computer Science and Applications (Montreal, QC).

[13] S. C. Milne. A $q$-analog of restricted growth functions, Dobinski's equality, and Charlier polynomials. Trans. Amer. Math. Soc., 245:89-118, 1978.

[14] V. Reiner. Non-crossing partitions for classical reflection groups. Discrete Math., 177(13):195-222, 1997

[15] J. B. Remmel and A. T. Wilson. An extension of MacMahon's equidistribution theorem to ordered set partitions. J. Combin. Theory Ser. A, 134:242-277, 2015.

[16] B. E. Sagan. A maj statistic for set partitions. European J. Combin., 12(1):69-79, 1991.

[17] R. Simion. Combinatorial statistics on noncrossing partitions. J. Combin. Theory Ser. A, 66(2):270-301, 1994

[18] E. Steingrimsson. Statistics on ordered partitions of sets. arXiv:math/0605670 [math.CO].

[19] M. Wachs and D. White. $p, q$-Stirling numbers and set partition statistics. J. Combin. Theory Ser. A, 56(1):27-46, 1991.

[20] D. White. Interpolating set partition statistics. J. Combin. Theory Ser. A, 68(2):262-295, 1994.

[21] A. T. Wilson. An extension of MacMahon's equidistribution theorem to ordered multiset partitions. Electron. J. Combin., 23(1):Paper 1.5, 21, 2016.

[22] J. Zeng and C. Zhang. A $q$-analog of Newton's series, Stirling functions and Eulerian functions. Results Math., 25(3-4):370-391, 1994. 